\title{
Extension of the Set of Complex Hadamard Matrices of Size 8
}

\author{
Wojciech T. Bruzda
}

Received: 10 January 2018 / Revised: 5 February 2018 / Accepted: 20 March 2018 /

Published online: 19 September 2018

(C) The Author(s) 2018

\begin{abstract}
A complex Hadamard matrix is defined as a matrix $H$ which fulfills two conditions, $\left|H_{j, k}\right|=1$ for all $j$ and $k$ and $H H^{*}=N \mathbb{I}_{N}$ where $\mathbb{I}_{N}$ is an identity matrix of size $N$. We explore the set of complex Hadamard matrices $\mathcal{H}_{N}$ of size $N=8$ and present two previously unknown structures: a one-parametric, non-affine family $T_{8}^{(1)}$ of complex Hadamard matrices and a single symmetric and isolated matrix $A_{8}^{(0)}$.
\end{abstract}

Keywords Complex Hadamard matrix $\cdot$ Non-affine family $\cdot$ Isolated matrix $\cdot$ Butson class

Mathematics Subject Classification $15 \mathrm{~A} 03 \cdot 65 \mathrm{~F} 25 \cdot 15 \mathrm{~B} 34 \cdot 05 \mathrm{~B} 20$

\section{Introduction}

The description of the set $\mathcal{H}_{N}$ of complex Hadamard matrices (CHMs) is exhausted for $N \in\{2,3,4,5\}[1,2]$, while beginning from $N=6$, general knowledge of the structure of $\mathcal{H}_{N}$ can be considered incomplete. CHMs and their subset containing only real $\mp 1$-entry objects are a subject of great interest in many fields of contemporary mathematics and physics. Let us only mention applications in quantum optics, telecommunication and signal processing, assistance in error correcting codes, background for equiangular lines, and many others [2-6].

Two matrices $H_{1}$ and $H_{2} \in \mathcal{H}_{N}$ are said to be equivalent if one of them can be expressed in terms of the other by means of two permutation matrices $P_{1}$ and $P_{2}$ and two unitary diagonal matrices $D_{1}$ and $D_{2}$ as $H_{1}=P_{1} D_{1} H_{2} P_{2} D_{2}$ $[2,7]$. In general, the recognition of equivalence is not trivial and requires additional tools such as Haagerup invariants [2] or matrix fingerprint [8]. Another signature assigned to a matrix is its defect [7,9]. The defect of a unitary matrix $d(H)$ tells us what is the largest possible size of a smooth family of CHMs stemming from $H$. This one-way criterion is useful when searching for isolated matrices for which the defect vanishes. There are two possible types of CHM families: affine and non-affine. In the first case, the particular constant entries in given matrix $H$ can be extended to complex variables so that their phases vary linearly as in the following example of the 4-dimensional Fourier matrix [1]

W. T. Bruzda $(\varangle)$

Institute of Physics, Jagiellonian University, Kraków, Poland

e-mail: w.bruzda@uj.edu.pl 
$F_{4}=\left[\begin{array}{rrrr}1 & 1 & 1 & 1 \\ 1 & i & -1 & -i \\ 1 & -1 & 1 & -1 \\ 1 & -i & -1 & i\end{array}\right] \mapsto\left[\begin{array}{rrrr}1 & 1 & 1 & 1 \\ 1 & i e^{i \alpha} & -1 & -i e^{i \alpha} \\ 1 & -1 & 1 & -1 \\ 1 & -i e^{i \alpha} & -1 & i e^{i \alpha}\end{array}\right]: \alpha \in[0,2 \pi)$

The second case involves non-linear formulas for the phases of the variable entries $[7,12]$.

Every CHM can be transformed by an equivalence preserving operation into the form in which its first row and its first column has only ones. The remaining $(N-1) \times(N-1)$ elements shall be called the core of the matrix. All matrices in this paper are worked out in the above dephased form.

A special subclass of CHMs is called Butson type matrices [10,11], denoted by $B H(N, q) \subset \mathcal{H}_{N}$, with the property that each entry of $H \in B H(N, q)$ is the $q$ th root of unity, $\left(H_{j, k}\right)^{q}=1$ for any $j$ and $k$. For instance, the standard class of real Hadamard matrices of size $N$ forms the class $B H(N, 2)$, while the Fourier matrix $F_{N}$ of order $N$ belongs to the set $B H(N, N)$.

The notation adopted in this work is entirely taken from the on-line CHM catalogue [12] based on [7] and, to save space, we use the symbol $[\beta]$ as a shortcut for the exponent expression $\exp (i \pi \beta)$.

\section{Looking for a New Matrix $H \in \mathcal{H}_{8}$}

Searching for new CHMs supports the description of the set $\mathcal{H}_{N}$ and provides better understanding of its elements. Moreover, additional profit from having new examples of CHMs is extension of the area for searching for new mutually unbiased pairs of Hadamard matrices $H_{1}$ and $H_{2}$ such that $H_{1}^{*} H_{2} / \sqrt{N} \in \mathcal{H}_{N}$, which is directly related to mutually unbiased bases [13-15].

Looking for possibly new complex Hadamard matrices, the following procedure was used. Let us take any dephased matrix $H$ with unimodular entries $H_{j, k}=\left[\varphi_{j, k}\right]$ for $\varphi_{j, k} \in[0,2)$. Define a target function $Z(H) \equiv$ $\left\|H H^{*}-N \mathbb{I}_{N}\right\|_{\mathrm{F}}$, where the Frobenius norm of matrix is applied, $\|X\|_{\mathrm{F}}^{2}=\operatorname{tr} X X^{*}$. Using a random-walk procedure over the core phases we try to minimize $Z(H)$ and for $Z(H)<10^{-16}$ we can stop having a matrix $\mathrm{H}$ that belongs to $\mathcal{H}_{N}$ with respect to a given precision. Such raw matrices are ready for further analytical processing. The most interesting outcomes show up when only a subset of core phases undergoes variation while the other elements are fixed to certain values. The algorithm is very fast and even using an average desktop PC for $N<9$ one can obtain results within seconds.

The case of $N=6$, however without any formal proof of completeness, seems to be already understood and it is conjectured that the families $G_{6}^{(4)}$ of Szöllősi [16] and $K_{6}^{(3)}$ of Karlsson [17] along with the isolated Tao matrix $S_{6}^{(0)}[18,19]$ constitute the set in which any $H \in \mathcal{H}_{6}$ is included. The set $\mathcal{H}_{7}$ consists of several isolated matrices: $F_{7}^{(0)}$ [7], $C_{7}^{(0)}[2,20]$ and $Q_{7}^{(0)}$ [21] together with one affine family $P_{7}^{(1)}$ [22]. The investigation of this case is far from being closed but proved to be hard for any numerical attempts. Hence, in this short paper, we focus on the next promising case for $N=8$ and, as a consequence, we present previously unknown:

- a 1-parametric, non-affine family $T_{8}^{(1)}$ of CHMs that contains Butson $B H(8,20)$ matrices,

- an isolated matrix $A_{8}^{(0)}$ of a non-Butson type.

\section{Non-affine Family $T_{8}^{(1)}$ of $N=8$ Complex Hadamard Matrices}

Let us start with the matrix $T_{8} \in \mathcal{H}_{8}$ that was found numerically (see Sect. 2) and then expressed in the analytic form using symmetric patterns in the elements and phases of the core and orthogonality constraints with respect to the rows and columns. Define a constant matrix: 


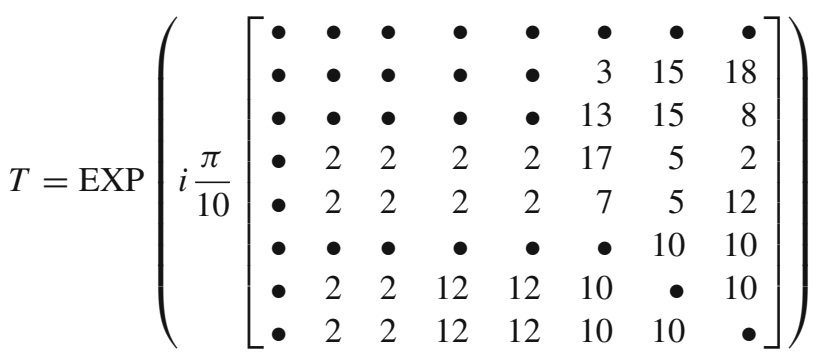

where $T=\operatorname{EXP}(A)$ denotes the entrywise exponent of a matrix, $T_{j, k}=\exp \left(A_{j, k}\right)$ and $\bullet$ replaces zero. Furthermore, we shall use the matrix

$T_{c}=\left[\begin{array}{rrrrrrrr}1 & 1 & 1 & 1 & 1 & 1 & 1 & 1 \\ 1 & c_{3} & c_{2} & c_{1} & -i / c_{1} & 1 & 1 & 1 \\ 1 & -c_{1} & i / c_{1} & -i / c_{2} & -i / c_{3} & 1 & 1 & 1 \\ 1 & c_{2} & c_{3} & -i / c_{1} & c_{1} & 1 & 1 & 1 \\ 1 & -i / c_{1} & c_{1} & i / c_{3} & i / c_{2} & 1 & 1 & 1 \\ 1 & e^{i \alpha} & -e^{i \alpha} & -e^{-i \alpha} & e^{-i \alpha} & 1 & 1 & 1 \\ 1 & e^{i \alpha} & -e^{i \alpha} & -e^{-i \alpha} & e^{-i \alpha} & 1 & 1 & 1 \\ 1 & 1 & 1 & 1 & 1 & 1 & 1 & 1\end{array}\right]$.

with

$$
\begin{aligned}
& c_{1}=\frac{1}{4} \sqrt{8-\sqrt{50-6 \sqrt{5}}}+i \frac{1}{4} \sqrt{8+\sqrt{50-6 \sqrt{5}}} \\
& c_{2}=\frac{i-1-\left[\frac{3}{10}\right]+\left[\frac{4}{5}\right]-c_{1}+i / c_{1}}{1-i c_{1}^{2}} \\
& c_{3}=-i c_{2} c_{1}^{2}
\end{aligned}
$$

where $\alpha=-\operatorname{arccot}(1-\sqrt{5})$ and $[\beta]=\exp (i \pi \beta)$. This allows us to define the following complex Hadamard matrix $T_{8}=T_{c} \circ T$ where $\circ$ is the entrywise product of matrices.

One can check that the defect $d\left(T_{8}\right)=3$ which is an indication that the matrix can be a part of a family of inequivalent CHMs. Moreover, the structure of the three last columns resembles the form of a Butson type object which might suggest that a Butson matrix lies on a trajectory of the orbit stemming from a given matrix and some entries $\left(T_{8}\right)_{j, k}$ for $2 \leqslant j \leqslant 7$ and $2 \leqslant k \leqslant 5$ could be functionally dependent. Indeed, when one assigns to two particular elements $u(\mu)=\left(T_{8}\right)_{7,2}$ and $-u(\mu)=\left(T_{8}\right)_{7,3}$ a value $\mu \in[0,1 / 2]$ for $u=[\mu]$, having the last three columns fixed, there always can be found a CHM with the second row of the form

$$
\left[1, x, y, z,-\frac{y z}{x},\left[\frac{3}{10}\right],-i,\left[\frac{18}{10}\right]\right] \text {. }
$$


Again, using symmetries amongst phases and orthogonality constraints, one recovers a full matrix in the form of

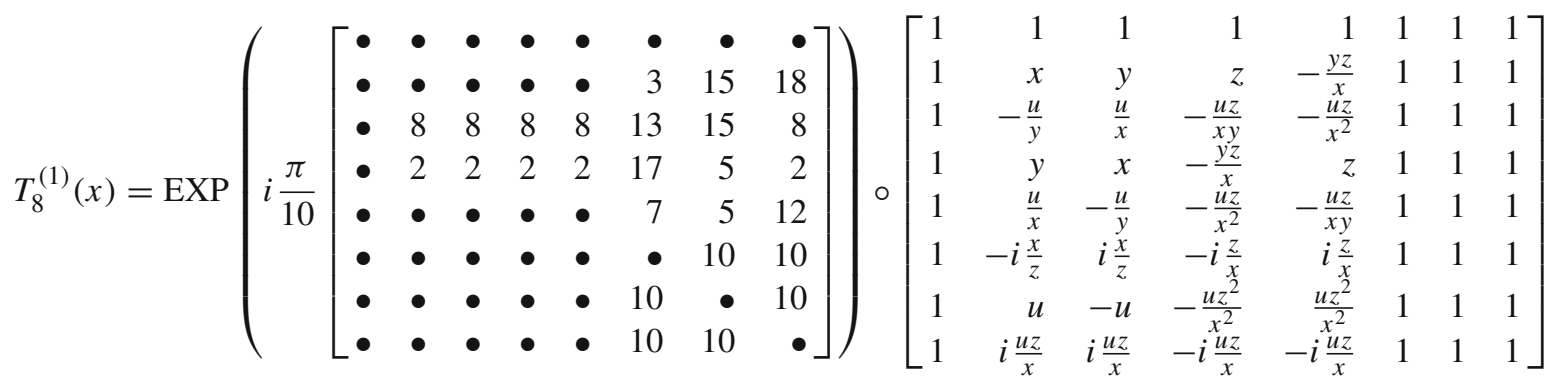

where

$$
\begin{aligned}
& \chi_{1}=5-3 \sqrt{5}+4 \sqrt{5-2 \sqrt{5}}+(1+i) \frac{1}{x}+\zeta_{2}, \\
& \chi_{2}=45-9 i-(17-7 i) \sqrt{5}-2 \sqrt{620-500 i-(285-234 i) \sqrt{5}}-(1+i) \frac{\chi_{1}}{x}, \\
& \chi_{3}=10 i-10+(6-2 i) \sqrt{5}+4 \sqrt{10-10 i-(5-4 i) \sqrt{5}}+(1+i) \frac{\chi_{2}}{x}, \\
& \chi_{4}=\sqrt{5}-1+i \sqrt{2} \sqrt{5+\sqrt{5}}+(1+i) \frac{\chi_{3}}{x}, \\
& \chi_{5}=(1+i) \sqrt{1-\sqrt{5}+\zeta_{4}}-(1-i)\left(2 x^{2}+\left(\sqrt{\chi_{4}}\right)^{*}\right)-x\left(1-4 i+\sqrt{5}+\zeta_{1}\right), \\
& \chi_{6}=\sqrt{5}-1-i-\sqrt{5-2 \sqrt{5}}+(1-i) x, \\
& y=\frac{1}{4} \frac{\chi_{5}}{\chi_{6}} \\
& z=\frac{x}{x-y}\left((1+i)\left[\frac{4}{5}\right]-x-y-1+i\right),
\end{aligned}
$$

with $\zeta_{1}=-i \sqrt{10-2 \sqrt{5}}, \zeta_{2}=-\zeta_{1}$ and $\zeta_{4}=i \sqrt{10+2 \sqrt{5}}$ being the roots of the polynomial equation $\zeta^{4}+$ $20 \zeta^{2}+80=0$ and eventually

$u=\frac{i}{z} \frac{x^{2}-z x+x y+y z}{1+i-\left[\frac{3}{10}\right]-\left[\frac{4}{5}\right]}$.

Unimodular number $x=[\gamma]$ is a free non-affine parameter. ${ }^{1}$ In order to assure unimodularity of all other entries one can select phase $\gamma$ from the range ${ }^{2} \gamma \in[2 / 5,4 / 5] \cup[1,7 / 5]$.

\footnotetext{
${ }^{1}$ Ready-to-use scripts in Matlab and Mathematica are available on-line [12].

2 The actual range for $\gamma$ is slightly broader, however, it does not reveal any more information.
} 
As presumed, there are two Butson matrices that can be found inside the orbit $T_{8}^{(1)}$. For $\gamma \in\{1 / 2,1,13 / 10\}$ one obtains three matrices equivalent to $B_{8 a} \in B H(8,20)$ with defect 7 ,

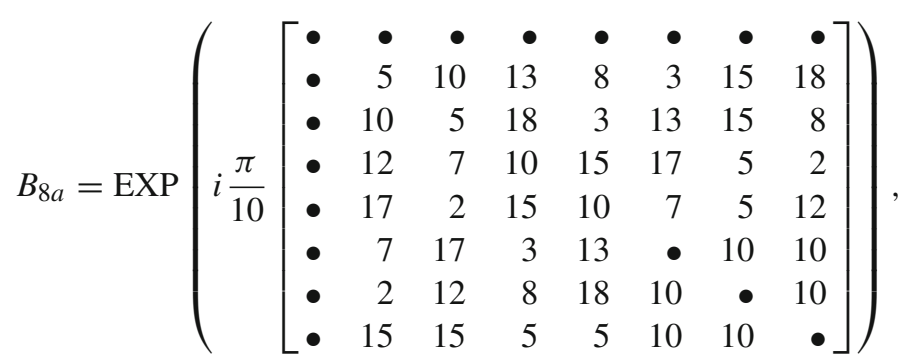

while for $\gamma=4 / 5$ there exists another Butson matrix $B_{8 b} \in B H(8,20)$ with defect 11 ,

$$
B_{8 b}=\operatorname{EXP}\left(i \frac{\pi}{10}\left[\begin{array}{rrrrrrrr}
\bullet & \bullet & \bullet & \bullet & \bullet & \bullet & \bullet & \bullet \\
\bullet & 8 & 10 & 13 & 5 & 3 & 15 & 18 \\
\bullet & 18 & 10 & 3 & 5 & 13 & 15 & 8 \\
\bullet & 12 & 10 & 7 & 15 & 17 & 5 & 2 \\
\bullet & 10 & \bullet & \bullet & 10 & \bullet & 10 & 12 \\
\bullet & 10 & \bullet & 10 & \bullet & 10 & \bullet & 10 \\
\bullet & \bullet & \bullet & 10 & 10 & 10 & 10 & \bullet
\end{array}\right]\right)
$$

It is likely that the above Butson matrices have not yet appeared in the literature, as they are not listed in the on-line catalogues [12,23,24].

No other Butson matrices intersect the orbit $T_{8}^{(1)}$ which implies that this family does not affect any other matrices of size 8 known up to date: $F_{8}^{(5)}$ [7], $S_{8}^{(4)}$ [25], $D_{8}^{(4)}$ [26] or $V_{8}^{(0)}$ [27].

\section{New Isolated Matrix $A_{8}^{(0)}$}

Using the random walk procedure with additional constraint for $H$ to be symmetric we were able to find the following matrix:

$$
A_{8}^{(0)}=\left[\begin{array}{rrrrrrrr}
1 & 1 & 1 & 1 & 1 & 1 & 1 & 1 \\
1 & -a^{2} & -1 & a^{2} & a & -a & -a & a \\
1 & -1 & -1 & -a & 1 / a & 1 & -1 / a & a \\
1 & a^{2} & -a & a & -1 & a & -a & -a^{2} \\
1 & a & 1 / a & -1 & 1 & -1 & -1 / a & -a \\
1 & -a & 1 & a & -1 & 1 / a & -1 / a & -1 \\
1 & -a & -1 / a & -a & -1 / a & -1 / a & 1 & -a \\
1 & a & a & -a^{2} & -a & -1 & -a & a^{2}
\end{array}\right],
$$

with $a=\frac{1}{3}+i \frac{2 \sqrt{2}}{3}$ being a solution of the equation $3 a^{2}-2 a+3=0$.

The matrix is not of the Butson type for its elements are incommensurate with any root of unity. Its defect value is 0 thus the matrix is isolated and no family can stem from it. Obviously, this matrix does not touch any objects discussed above. 


\section{Concluding Remarks}

In this paper we have found two previously unknown objects from the set of complex Hadamard matrices $\mathcal{H}_{8}$ of size 8 - the isolated matrix $A_{8}^{(0)}$ which is not of the Butson type and 1-parametric, non-affine family of inequivalent matrices $T_{8}^{(1)}$. This does not provide complete classification of the case $N=8$ but hopefully will help to develop another ideas to describe a full structure of $\mathcal{H}_{8}$. Generic defect of $T_{8}^{(1)}$ is 3 so it might be a part of a higher dimensional structure. An imposing question is whether there exist any other 8-dimensional families which possibly connect some of currently known non-isolated elements of $\mathcal{H}_{8}$.

Acknowledgements The author is indebted to Dardo Goyeneche, Ferenc Szöllősi, Konrad Szymański and Karol Życzkowski for helpful discussions. Furthermore, the author would like to thank the anonymous referee for several remarks that allowed to improve the paper significantly. Financial support by Narodowe Centrum Nauki under the grant number DEC-2015/18/A/ST2/00274 is gratefully acknowledged.

Open Access This article is distributed under the terms of the Creative Commons Attribution 4.0 International License (http:// creativecommons.org/licenses/by/4.0/), which permits unrestricted use, distribution, and reproduction in any medium, provided you give appropriate credit to the original author(s) and the source, provide a link to the Creative Commons license, and indicate if changes were made.

\section{References}

1. Craigen, R.: Equivalence classes of inverse orthogonal and unit Hadamard matrices. Bull. Austral. Math. Soc. 44, 109-115 (1991)

2. Haagerup, U.: Orthogonal Maximal Abelian $*$-Subalgebras of the $n \times n$ Matrices and Cyclic $n$-roots, pp. 296-322. International Press, Cambridge (1996)

3. Seberry, J., Wysocki, B.J., Wysocki, T.: On some applications of Hadamard matrices. Metrica 62, 221-239 (2005)

4. Werner, R.: All teleportation and dense coding schemes. J. Phys. A 34, 7081-7094 (2001)

5. Heng, I., Cooke, C.H.: Error correcting codes associated with complex Hadamard matrices. Appl. Math. Lett. 11, 77-80 (1998)

6. Godsil, C.D., Roy, A.: Equiangular lines, mutually unbiased bases, and spin models. Eur. J. Comb. 30, 246-262 (2009)

7. Tadej, W., Życzkowski, K.: A concise guide to complex Hadamard matrices. Open Syst. Inf. Dyn. 13, 133-177 (2006)

8. Szöllősi, F.: Exotic complex Hadamard matrices and their equivalence. Cryptogr. Commun. 2, 187-198 (2010)

9. Tadej, W., Życzkowski, K.: Defect of a unitary matrix. Linear Algebra Appl. 429(2-3), 447-481 (2008)

10. Butson, A.T.: Generalized Hadamard matrices. Proc. AMS 13, 894-898 (1962)

11. Butson, A.T.: Relations among generalized Hadamard matrices, relative difference sets, and maximal length linear recurring sequences. Can. J. Math. 15, 42-48 (1963)

12. Bruzda, W., Tadej, W., Życzkowski, K.: On-line Catalogue of Complex Haramard Matrices. http://chaos.if.uj.edu.pl/ karol/ hadamard/. Accessed 25 Feb 2018

13. Zauner, G.: Ph.D. Thesis, University of Wien (1999)

14. Wootters, W., Fields, B.: Optimal state-determination by mutually unbiased measurements. Ann. Phys. 191, 363-381 (1989)

15. Grassl, M.: On SIC-POVMs and MUBs in Dimension 6 (2009). arXiv:quant-ph/0406175

16. Szöllősi, F.: Complex Hadamard matrices of order 6: a four-parameter family. J. Lond. Math. Soc. 85(3), 616-632 (2012)

17. Karlsson, B.R.: Three parameter complex Hadamard matrices of order 6. Linear Algebra Appl. 434, $247-258$ (2011)

18. Moorhouse, G.E.: The 2-Transitive Complex Hadamard Matrices (2001). http://citeseerx.ist.psu.edu/viewdoc/summary?doi=10.1. 1.32.4884. Accessed 25 Feb 2018

19. Tao, T.: Fuglede's conjecture is false in 5 and higher dimensions. Math. Res. Lett. 11, 251-258 (2004)

20. Björck, G., Fröberg, R.: A faster way to count the solutions of inhomogeneous systems of algebraic equations, with applications to cyclic $n$-roots. J. Symb. Comput. 12, 329-336 (1991)

21. Szöllősi, F.: Ph.D. Thesis, CEU Hungary (2011)

22. Petrescu, M.: Ph.D. Thesis, UCLA (1997)

23. Lampio, P.H.J., Ostergård, P.R.J., Szöllősi, F.: Orderly Generation of Butson Hadamard Matrices (2017). arXiv:1707.02287

24. Lampio, P.H.J.: On-line Catalogue of Butson Matrices. https://wiki.aalto.fi/display/Butson/Butson+Home. Accessed 25 Feb 2018

25. Matolcsi, M., Réffy, J., Szöllősi, F.: Constructions of complex Hadamard matrices via tiling Abelian groups. Open Syst. Inf. Dyn. 14, 247-263 (2006)

26. Diţă, P.: Complex Hadamard Matrices from Sylvester Inverse Orthogonal Matrices (2010). arXiv:0901.0982v3

27. Elser, V.: Private communication (2011), http://chaos.if.uj.edu.pl/ karol/hadamard/?q=0804 